\title{
A numerical method for the semilinear stochastic transport equation
}

\author{
Hugo de la Cruz \\ Escola de Matemática Aplicada-FGV \\ Praia de Botafogo 190, RJ \\ E-mail: hugo.delacruz@fgv.br
}

\author{
Christian H. Olivera \\ IMECC UNICAMP \\ Barão Geraldo, Campinas, SP \\ E-mail: colivera@imecc.unicamp.br
}

\author{
Jorge P. Zubelli \\ IMPA - Instituto de Matemática Pura e Aplicada \\ Estrada Dona Castorina 110, RJ \\ E-mail: zubelli@impa.br
}

\begin{abstract}
We propose a new numerical method for the computer simulation of the semi-linear stochastic transport equation. Based on the stochastic characteristic method and the Local Linearization technique, we construct an efficient and stable method for integrating this equation. For this purpose, a suitable exponential-based approximation to the solution of an associated auxiliary random integral equation, together with a Padé method with scaling and squaring strategy are conveniently combined. Results on the convergence and stability of the suggested method and details on its efficient implementation are discussed.
\end{abstract}

keywords: Stochastic partial differential equations, numerical methods, stochastic transport equation, local linearization approach, exponential integrators

\section{Introduction}

In this paper we are concerned with the numerical integration of the semilinear stochastic transport equation.

$$
\left\{\begin{array}{l}
\frac{d}{d t} u(t, x)+b(t, x) \nabla u(t, x)+\sigma(t) \frac{d B_{t}}{d t} \nabla u(t, x)+G(t, x, u)=0, \\
u(0, x)=u_{0}(x),
\end{array}\right.
$$

where $B_{t}=\left(B_{t}^{1}, \ldots, B_{t}^{d}\right)$ is a $d$-dimensional Brownian Motion, $G(t, ., u) \in C^{1}\left(\mathbb{R}^{d}\right)$, the matrix $\sigma(t)=\operatorname{diag}\left(\sigma^{1}(t), \ldots, \sigma^{d}(t)\right)$ and the velocity $b(t, x) \in L^{2}\left([0, T], C^{m, \delta}\left(\mathbb{R}^{d}\right)\right), m \geq 3$. This equation arises by considering the velocity field of the deterministic semi-linear transport equation as $b_{i}(x, t)+\sum_{n=1}^{d} \sigma_{i j}(t) \frac{d B_{t}^{j}}{d t}$, that is, as the sum of a random field and a spatially dependent white noise. Formally, the equation (1) is interpreted as the stochastic integral equation

$$
\left\{\begin{array}{l}
u(t, x)=u_{0}(x)-\int_{0}^{t} b(s, x) \nabla u(s, x) d s-\sum_{i=0}^{d} \int_{0}^{t}\left(\frac{\partial}{\partial x_{i}} u(s, x) \sigma^{i}(s)\right) \circ d B_{s}^{i}-\int_{0}^{t} G(s, x, u) d s, \\
u(0, x)=u_{0}(x),
\end{array}\right.
$$

where the stochastic integration is taken in the Stratonovich sense.

In applications, these kind of equations arises as a prototype model in understanding a wide variety of phenomena. It appears in modeling transport in porous media, suspended sediment transport in open-channel flows, advective transport of substances, waves motions in random media and from turbulent transport theory, $u(t, x)$ being the concentration of a passive substance convected by a turbulent fluid, such as smoke in turbulent air. 
The existence, uniqueness, and properties of the solutions of such equations have been well studied for the case of classical solutions in [8] and [9] (see also [5]) via the stochastic characteristic method. Since in general it is not possible to derive an analytical solution for (1), the accurate simulation of this equation is essential in order to get a better understanding and a valuable information of the phenomena in study.

In the scenario of the numerical simulation of transport equation there are some related works but with focus on the numerical solution of the unidirectional random transport equation, which essentially is a deterministic transport equation with the velocity and/or the initial condition been stochastic input parameters (see for instance [3], [4], [11]). However, as far as we know, the reliable simulation of the stochastic -Stratonovich- transport equation (1) has not been considered so far. In the present work we attempt to go forward in this direction by proposing a new numerical method for the computational simulation of (1).

Our aim here is to exploit the stochastic characteristics method and numerical integration of stochastic and random differential equations together with the local linearization technique [2] to construct an exponential-based integrator to (1). The idea consist in using the stochastic characteristic method to get a representation for $u(t, x)$ through the solution of an associated stochastic differential equation (SDE), a random differential equation (RDE) and a corresponding backward random equation (which can be conveniently transformed to a random integral equation (RIE) in the forward sense). From a computational point of view the main difficulty is to construct an efficient integrator for this RIE. For this, we elaborate the following strategy: at each time interval of integration, the vector field is locally approximated through a first order Taylor expansion and the stochastic term (which has low regularity) is approximated by a polygonal obtained by a suitable interpolation. In this way successive Caratheodory RDEs are obtained and their solutions can be explicitly expressed in terms of a single matrix exponential times a vector. This exponential representation is a key point in the design of feasible computational algorithms implementing the method. The reason is that the involved matrix has a particular structure that allows to derive an algorithm based on the Padé method with scalingsquaring strategy in such a way that the overall computational saving achieved are significant and consequently resulting in accurate and stable numerical schemes. It can be proved that the rate of convergence of the method obtained in this way, is essentially determined by the moduli of continuity of Wiener processes.

\section{The proposed method}

We begin this section by presenting the so called stochastic characteristics method, that will be used as a key tool to design our method. The stochastic characteristic method says (see [9]) that for each $(T, \mathbf{x})$ the solution $u$ of $(1)$ can be represented as

$$
u(T, \mathbf{x})=Z_{T}\left(u_{0}\left(X_{0, T}^{-1}(\mathbf{x})\right)\right)
$$

where $Z_{t}(r)$ satisfies the RDE

$$
Z_{t}=r+\int_{0}^{t} G\left(s, X_{s}(\mathbf{x}), Z_{s}\right) d s
$$

$X_{t}(x)$ satisfies the additive noise $\mathrm{SDE}$

$$
X_{t}=\mathbf{x}+\int_{0}^{t} b\left(s, X_{s}\right) d s+\int_{0}^{t} \sigma(s) \circ d B_{s}
$$



RIE

and the inverse stochastic flow satisfies $X_{0, \mathbf{T}}^{-1}(\mathbf{x})=\mathbf{R}(T)$, where $\mathbf{R}(t)$ is the solution of the

$$
\mathbf{R}(t)=\mathbf{x}-\int_{0}^{t} \mathbf{f}(s, \mathbf{R}(s)) d s-\left(\xi_{T}-\xi_{T-t}\right), \quad \xi_{t}=\int_{0}^{t} \sigma(s) \circ d B_{s},
$$

with $\mathbf{f}(s, \mathbf{R})=b(T-s, \mathbf{R})$. Thus, our numerical integrator will be obtained by combining numerical integrators for (3), (2) and (4). Since the solution of (3) can be computed independently of (2), it allows to work with (2) as a RDE with the stochastic process $X_{s}$ known in any desirable point, in particular in the discretization points to be used by the numerical method solving (2).

Let $(t)_{h}=\left\{t_{n}: n=0,1, \ldots, N\right\}$ be a partition of the time interval $\left[t_{0}, T\right]$ with equidistant stepsize $h$. For the stable integration of equation (3) we will use the recent Local Linearization methods proposed in [2], so we will consider the method

$$
\left.\mathbf{y}_{n+1}=\mathbf{y}_{n}+\left[\begin{array}{ll}
\mathbf{I}_{d \times d} & \mathbf{0}_{d \times 2}
\end{array}\right] e^{\left(\begin{array}{ccc}
b_{x} & b_{t} & b \\
0 & 0 & 1 \\
0 & 0 & 0
\end{array}\right) h\left[\mathbf{0}_{1 \times d+1} 1\right.}\right]^{\top}+e^{b_{x} h} \sigma(t)\left(\Delta B^{(n+1)}\right) .
$$

where $\Delta B^{(n+1)}=B\left(t_{n+1}\right)-B\left(t_{n}\right)$ and $b_{x}, b_{t}, b$ are evaluated in $\left(t_{n}, \mathbf{y}_{n}\right)$. For the stable integration of (2) we can use any of the well known methods proposed in [1] and [7]. Specifically, when using the LL method proposed in [1] we have,

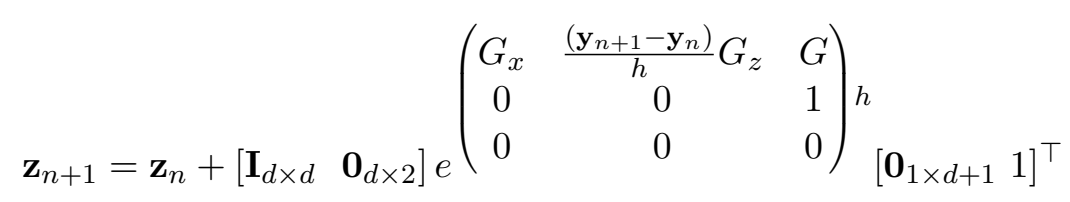

where $G_{x}, G_{z}, G$ are evaluated in $\left(t_{n}, \mathbf{y}_{n}, \mathbf{z}_{n}\right)$.

As mentioned in the introduction, the main difficulty is to elaborate an efficient and stable integrator for the IRE (4). In that follows we will concentrate in devising a numerical integrator for this equation.

\subsection{An integrator for the RIE}

Let us consider the partition $(t)_{h}$. Starting from the initial value $\mathbf{R}_{0}=\mathbf{R}\left(t_{0}\right)$, the approximations $\left\{\mathbf{R}_{i}\right\}$ to $\left\{\mathbf{R}\left(t_{i}\right)\right\},(i=1,2, \ldots, N)$ are obtained recursively as follows.

For each time interval $\mathbf{I}_{n}=\left[t_{n}, t_{n+1}\right]$ we consider the random local problem

$$
\mathbf{R}(t)=\mathbf{R}_{n}-\int_{t_{n}}^{t} \mathbf{f}(s, \mathbf{R}(s)) d s+\xi_{T-t_{n}}-\xi_{T-t} .
$$

Then, the idea is to get an approximation of $\mathbf{R}\left(t_{n+1}\right)$, through the solution of the auxiliary random equation resulting from approximating $\mathbf{f}$ and the stochastic increment in (7). For this, let's consider $\bar{h}=h^{\gamma}$ with $\gamma>4$ and such that $h^{1-\gamma} \in \mathbb{N}$ (we need to take $\bar{h}$ in this way in order to guaranty the convergence of the method we are constructing here) and let $\left(t_{n}\right)_{\bar{h}}=\left\{t_{n}^{(i)}: t_{n}^{(i)}=t_{n}+i \bar{h}, i=0,1, \ldots,\left[h^{1-\gamma}\right]+1\right\}$ a partition of $\mathbf{I}_{n}$. For $t \in \mathbf{I}_{n}$, let $k$ such that $t_{n}^{(k)} \leq t<t_{n}^{(k+1)}$, then by a linear interpolation to $\xi_{T-t}$ in $\left[t_{n}^{(k)}, t_{n}^{(k+1)}\right]$ we have

$$
\xi_{T-t_{n}}-\xi_{T-t} \approx\left(\xi_{T-t_{n}}-\xi_{T-t_{n}^{(k)}}\right)+\frac{\xi_{T-t_{n}^{(k)}}-\xi_{T-t_{n}^{(k+1)}}}{\bar{h}}\left(t-t_{n}^{(k)}\right) .
$$

Hence, by using the approximations above and from a first order Taylor expansion of $\mathbf{f}$, it follows that $\mathbf{R}(t)$ is differentiable for $t \in\left\{\mathbf{I}_{n} \backslash\left(t_{n}\right)_{\bar{h}}\right\}$ and satisfies the (pathwise) Caratheodory differential equation

$$
\mathbf{R}^{\prime}(t)=\mathbf{A}_{n} \mathbf{R}(t)+\mathbf{b}_{n} t+\mathbf{c}_{n}^{k},
$$


where

$$
\begin{aligned}
\mathbf{A}_{n} & =-\mathbf{f}_{\mathbf{x}}\left(t_{n}, \mathbf{R}_{n}\right), \\
\mathbf{b}_{n} & =-\mathbf{f}_{\mathbf{t}}\left(t_{n}, \mathbf{R}_{n}\right), \\
\mathbf{c}_{n}^{k} & =\mathbf{f}\left(t_{n}, \mathbf{R}_{n}\right)-\mathbf{A}_{\mathbf{n}} \mathbf{R}_{\mathbf{n}}-\mathbf{b}_{\mathbf{n}} t_{n}+\sum_{k=0}^{h^{1-\gamma}} \frac{\xi_{T-t_{n}^{(k)}-\xi_{T-t_{n}^{(k+1)}}}}{\bar{h}} \mathbf{1}_{\left[t_{n}^{(k)} t_{n}^{(k+1)}\right]}(t) .
\end{aligned}
$$

Since the inhomogeneity in (8) is discontinuous in $\left\{t_{n}^{(k)}\right\}$, the solution at $t=t_{n+1}$ is obtained recursively from $\mathbf{R}\left(t_{n}^{(k)}\right)$ by solving (8) with initial condition $\mathbf{R}\left(t_{n}^{(k)}\right)$ in $t=t_{n}^{(k)}$.

Thus,

$$
\mathbf{R}\left(t_{n}^{(k+1)}\right)=\mathbf{R}\left(t_{n}^{(k)}\right)+\int_{0}^{\bar{h}} e^{\mathbf{A}_{n}(\bar{h}-s)}\left(\mathbf{b}_{n} s+\mathbf{d}_{n}^{k}\right) d s:=\varphi\left(\mathbf{R}\left(t_{n}^{(k)}\right)\right),
$$

where $\mathbf{d}_{n}^{k}=\mathbf{c}_{n}^{k}+\mathbf{A}_{n} \mathbf{R}\left(t_{n}^{(k)}\right)+\mathbf{b}_{n} t_{n}^{(k)}$.

As we want to approximate $\mathbf{R}\left(t_{n+1}\right)$, we conclude that the numerical integrator for (4) is given by concatenating the $\left[h^{1-\gamma}\right]+1$ iterations of the function $\varphi$. That is

$$
\mathbf{R}_{n+1}=\varphi^{\left(h^{1-\gamma}\right)}\left(\mathbf{R}_{n}\right),
$$

for $n=0,1, \ldots, N-1$ with $\mathbf{R}\left(t_{0}\right)=\mathbf{R}_{0}$ and $\gamma \geq 4$.

An important problem in the evaluation of (10) is the efficient and stable computation of $\varphi$. A naive way to do this is through the explicit computation of the integral defining $\varphi$. However, this procedure might eventually fail since it is not computationally feasible in case of singular or nearly singular matrices $\mathbf{A}_{n}$ (see e.g. comments in [2]). In the next section we will propose an efficient algorithm for computing $\mathbf{R}_{n+1}$. Concerning the convergence and velocity of convergence of the proposed method we have the following theorem.

Theorem: Let's suppose that the moduli of continuity of $\xi$ satisfies that $\varpi_{\xi}(\bar{h})=O\left(\bar{h}^{\beta}\right)$ and let $\gamma \beta \geq 2$ and $p=\min (\gamma \beta, 3)$. Then the numerical integrator (10) is almost surely globally convergent and we have that with probability one $\sup _{n}\left\|\mathbf{R}\left(t_{n}\right)-\mathbf{R}_{n}\right\|=O\left(h^{p-1}\right)$.

Note that, because of the moduli of continuity of the Brownian is $\frac{1}{2}-\varepsilon$, we will have a convergent method just by taking $\gamma>4$.

\subsection{Implementation details}

In this section an efficient computational algorithm to implement (10) is provided. The initial key idea is that remarkably $\mathbf{R}\left(t_{n}^{(k+1)}\right)$ in (9) can be represented in terms of a single appropriated exponential of a matrix. That is

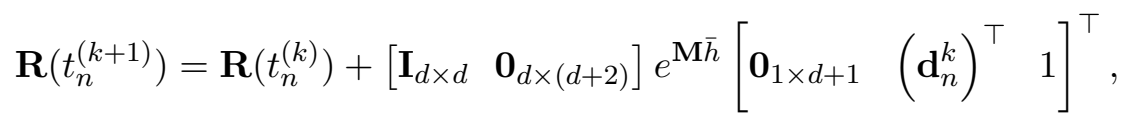

with

$$
\mathbf{M}=\left(\begin{array}{cccc}
\mathbf{A}_{n} & \mathbf{b}_{n} & \mathbf{I}_{d \times d} & \mathbf{0}_{d \times 1} \\
\mathbf{0}_{1 \times d} & 0 & \mathbf{0}_{1 \times d} & 1 \\
\mathbf{0}_{d \times d} & \mathbf{0}_{d \times 1} & \mathbf{0}_{d \times d} & \mathbf{0}_{d \times 1} \\
\mathbf{0}_{1 \times d} & 0 & \mathbf{0}_{1 \times d} & 0
\end{array}\right) \bar{h} .
$$

Thus, the numerical implementation of $\mathbf{R}\left(t_{n}^{(k+1)}\right)$ is reduced to the use of a algorithm to compute exponential of matrices (See [10] for a review). In particular, those algorithms based on the rational $(p, q)$-Padé approximation $(p \leq q \leq p+2)$ provide stable approximations. However, because of the size of the matrix $\mathbf{M}$, a straightforward implementation of the Padé method could be prohibitively expensive. In the rest of this subsection we propose an algorithm that alleviates significantly the computational burden. Our key idea is to exploit the special structure of the matrix $\mathbf{M}$ and to adapt conveniently the Padé method with "scaling and squaring" strategy in such a way that the computational saving achieved are significant. 


\subsubsection{The Adapted Padé algorithm for computing $e^{\mathrm{M} \bar{h}}$}

Let's define $\mathbf{C}=\mathbf{M} \bar{h}$. Let $k$ the minimum integer such that $\left\|\frac{\mathbf{C}}{2^{k}}\right\|<\frac{1}{2}$ and the coefficients $c_{j}=\frac{(2 q-j) ! q !}{(2 q) ! j !(p-j) !}(j=0, \ldots, q), \alpha_{j}=c_{j}\left(\frac{\bar{h}}{2^{k}}\right)^{j}$, then $e^{\mathbf{M} \bar{h}} \approx\left(\mathbf{P}_{q}\left(\frac{\mathbf{C}}{2^{k}}\right)\right)^{2^{k}}$ where $\mathbf{P}_{q}\left(\frac{\mathbf{C}}{2^{k}}\right)$ has the particular form

$$
\mathbf{P}_{q}\left(\frac{\mathbf{C}}{2^{k}}\right)=\left(\begin{array}{cccc}
\mathbf{U} 1 & \mathbf{U} 2 & \mathbf{U} 3 & \mathbf{U} 4 \\
\mathbf{0}_{1 \times d} & 1 & \mathbf{0}_{1 \times d} & \alpha_{1} \\
\mathbf{0}_{d \times d} & \mathbf{0}_{d \times 1} & \mathbf{I}_{d \times d} & \mathbf{0}_{d \times 1} \\
\mathbf{0}_{1 \times d} & 0 & \mathbf{0}_{1 \times d} & 1
\end{array}\right),
$$

with $\mathbf{U} 2=(\mathbf{U} 3) \mathbf{b}$ and the matrices $\mathbf{U} 1, \mathbf{U} 3$ and the vector $\mathbf{U} 4$ satisfying the systems of linear equations

$$
\begin{aligned}
& {\left[\mathbf{I}+\mathbf{A}\left(-\alpha_{1} \mathbf{I}+\mathbf{A} \overline{\mathbf{S}}\right)\right] \mathbf{U} 1=\left[\mathbf{I}+\mathbf{A}\left(\alpha_{1} \mathbf{I}+\mathbf{A S}\right)\right]} \\
& {\left[\mathbf{I}+\mathbf{A}\left(-\alpha_{1} \mathbf{I}+\mathbf{A} \overline{\mathbf{S}}\right)\right] \mathbf{U} 3=\left(\alpha_{1} \mathbf{I}+\mathbf{A S}\right)-\left(-\alpha_{1} \mathbf{I}+\mathbf{A} \overline{\mathbf{S}}\right),} \\
& {\left[\mathbf{I}+\mathbf{A}\left(-\alpha_{1} \mathbf{I}+\mathbf{A} \overline{\mathbf{S}}\right)\right] \mathbf{U} 4=\left[\mathbf{S}-2 \alpha_{1}\left(-\alpha_{1} \mathbf{I}+\mathbf{A} \overline{\mathbf{S}}\right)-\overline{\mathbf{S}}\right] \mathbf{b}}
\end{aligned}
$$

where $\mathbf{A}=\mathbf{A}_{n} \bar{h}, \mathbf{b}=\mathbf{b}_{n} \bar{h}, \mathbf{S}=\sum_{i=2}^{q} \alpha_{i} A^{i-2}$ and $\overline{\mathbf{S}}=\sum_{i=2}^{q}(-1)^{i-2} \alpha_{i} A^{i-2}$. Since remarkably the fundamental matrix of each system above is the same one (also note that has dimension $d$ in contrast with the order $2 d+2$ of $\mathbf{M}$ ), we can exploit this to yield significant improvements in the computational cost when solving these set of simultaneous equations. That is, we can get the $\mathbf{L U}$ decomposition of $\left[\mathbf{I}+\mathbf{A}\left(-\alpha_{1} \mathbf{I}+\mathbf{A} \overline{\mathbf{S}}\right)\right]$ and then use the standard procedure for obtaining the definitive solution of each system (see for instance [6]). Once that the $\mathbf{U} 1, \mathbf{U} 2, \mathbf{U} 3, \mathbf{U} 4$ are obtained, $\left(\mathbf{P}_{q}\left(\frac{\mathbf{C}}{2^{k}}\right)\right)^{2^{k}}$ has to be computed to conclude the scaling-squaring Padé method.

All in all, after some algebraic manipulations we get that

$$
\mathbf{R}\left(t_{n}^{(k+1)}\right)=\mathbf{R}\left(t_{n}^{(k)}\right)+\mathbf{L} \mathbf{d}_{n}^{k}+\mathbf{Q}
$$

where $\mathbf{L}=\left(\sum_{i=0}^{2^{k}-1}(\mathbf{U} \mathbf{1})^{i}\right)(\mathbf{U} \mathbf{3}) ; \quad \mathbf{Q}=\left(\sum_{i=0}^{2^{k}-1}(\mathbf{U} \mathbf{1})^{i}\right) \mathbf{U} 4+\alpha_{1}\left(\sum_{i=0}^{2^{k}-2}(m-1-i)(\mathbf{U} \mathbf{1})^{i}\right)(\mathbf{U} 3) \mathbf{b}$.

Note that $\mathbf{L}$ and $\mathbf{Q}$ remain the same in each subinterval $\left[t_{n}^{(k)} t_{n}^{(k+1)}\right]$; so these values are computed only once in each interval $\left[t_{n} t_{n+1}\right]$, in consequence the computational saving is significant.

Finally, we get that the approximation $\mathbf{R}_{n+1}$ to the solution $\mathbf{R}\left(t_{n+1}\right)$ of (4) is

$$
\begin{aligned}
R_{n+1} & =\left(\mathbf{I}+\mathbf{L} \mathbf{A}_{n}\right)^{h^{1-\gamma}} R_{n} \\
& +\sum_{i=0}^{h^{1-\gamma}}\left(\mathbf{I}+\mathbf{L} \mathbf{A}_{n}\right)^{i-1}\left(Q+L\left(\mathbf{f}\left(t_{n}, R_{n}\right)-A_{n} R_{n}\right)\right) h^{-\gamma}\left(\sum_{i=0}^{h^{1-\gamma}}\left(\mathbf{I}+\mathbf{L} \mathbf{A}_{n}\right)^{i-1} \Delta \xi^{(k-i)}\right) L,
\end{aligned}
$$

where $\Delta \xi$ are increments of the process $\xi$ on the partition $\left(t_{n}\right)_{\bar{h}}$.

Since $v=E\left(\left(\Delta \xi_{t_{n}^{k-i}}\right)^{2}\right)=\int_{t_{n}^{k-i}}^{t_{n}^{k-i}+\bar{h}} \sigma^{2}(s) d_{s}$, and $\Delta \xi_{t_{n}^{k-i}} \sim N(0, v)$, we have that we can simulate the stochastic increments by $\Delta \xi^{(k-i)}=\sqrt{v} \mathrm{~N}(0,1)$, where $\mathrm{N}(0,1)$ is the standard normal distribution. Se [2] for details.

\subsection{The definitive Algorithm}

In what follows, the definitive algorithm to approximate in $(T, \mathbf{x})$ the solution $u$ of $(1)$ is described. Once we set the stepsize $h$, and consider the partition $(\tau)_{h}=\left\{t_{0} \equiv 0, t_{1}, t_{2}, \ldots, t_{N}\right\}$ of $\left[\begin{array}{ll}0 & T\end{array}\right]$ (with $\left.t_{n+1}=t_{n}+h\right)$, the basic steps of the algorithm are:

- Set $\bar{h}=h^{\gamma}\left(\right.$ with $\gamma>4$ and $\left.h^{1-\gamma} \in \mathbb{N}\right)$. 
- Generate the independent random variables $\Delta \xi^{(j)}$ and the independent random variables $\Delta B^{(j)}$ involved in (13) and (5), respectively.

- From $R_{0}=\mathbf{x}$, repeat until $n=N$ the computation of $R_{n}$ in (13).

- From $\mathbf{y}_{0}=\mathbf{x}$, repeat until $n=N$ the computation of $\mathbf{y}_{n}$ in (5).

- From $\mathbf{z}_{0}=u_{0}\left(R_{N}\right)$, repeat until $n=N$ the computation of $\mathbf{z}_{n}$ in (6).

Then, $\mathbf{z}_{N}$ is the approximation to $u(T, \mathbf{x})$.

Note that If we are interested in computing $E(\phi(u(T, \mathbf{x})))$, where $\phi($.$) is a bounded func-$ tion, we apply Monte Carlo method. For this we need to apply the algorithm $M$ times to get approximate samples $\left\{\mathbf{z}_{N}^{[i]}\right\}_{i=1}^{M}$ from the distribution of $u(T, \mathbf{x})$. Our computed approximation to $E(\phi(u(T, \mathbf{x})))$ would then be the sample mean $\mu=\frac{1}{M} \sum_{i=1}^{M} \phi\left(\mathbf{z}_{N}^{[i]}\right)$.

\section{A numerical Test}

Figure 1 shows a simple example concerning the performance of the proposed methods when integrating the equation (1) on $\left[\begin{array}{ll}0 & 2\end{array}\right] \times\left[\begin{array}{ll}0 & 8\end{array}\right]$ with $b=x, \sigma(t)=2, G=x$, and initial condition $u_{0}(x)=e^{-2(x-2)^{2}}$. For this we consider the temporal and spacial discretization with $h=\Delta x=$ 0,05 and we use the proposed algorithm for computing $u(.,$.$) in each point of the grid. For$ reference, stochastic characteristic curves are also plotted. From this experiment (and others not reported here) we can confirm that the proposed algorithm is an attractive alternative for the purpose of achieving numerical stability with appropriated computational effort.

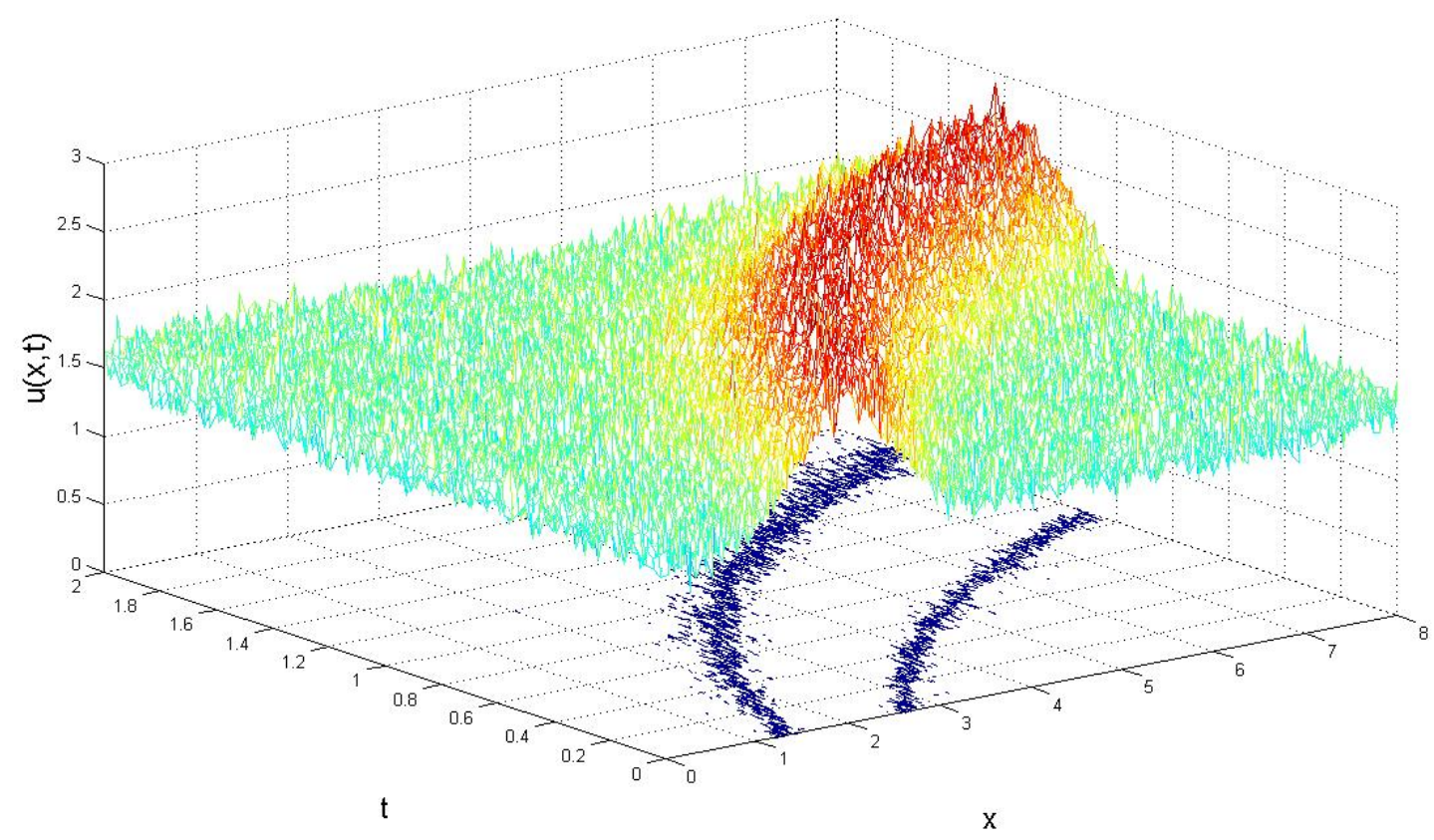

Figure 1: Computational integration of equation (1), on $\left[\begin{array}{ll}0 & 2\end{array}\right] \times\left[\begin{array}{ll}0 & 8\end{array}\right]$ with $b=x, \sigma(t)=2$, $G=x$, and initial condition $u_{0}(x)=e^{-2(x-2)^{2}}$, by the proposed method. The blue curves in the $x-t$ plane are characteristic curves. 


\section{Conclusions}

In this work we introduce an effective numerical integrator for the computer simulation of the semi-linear stochastic transport equation (1). For this we develop a computational method which is obtained via the solution of an suitable caratheodory-like RDE resulting for a associated RIE together with the numerical integration of SDEs and RDEs. It is important to note that thanks to the particular structure of the involved matrix in the proposed method, the computational burden is considerably reduced -from dimension $2 d+2$ to dimension $d$ - (via an adapted Padé method with scaling-squaring strategy) to the same computational load of solving simultaneous linear systems through a $\mathbf{L U}$ decomposition of a unique matrix. It is worth to note that the proposed method is well suited for parallel computing and since the computational cost scales with the dimension of the underlined original equation the approach has great potential even for very large simulation models.

\section{References}

[1] F. Carbonell, J.C. Jimenez, R.J. Biscay, H. de la Cruz, The Local Linearization method for numerical integration of random differential equations, BIT Num. Math, 45 (2005), 1-14.

[2] H. de la Cruz, R.J. Biscay, J.C. Jimenez, F. Carbonell, T. Ozaki, High Order Local Linearization methods: an approach for constructing A-stable high order explicit schemes for stochastic differential equations with additive noise, BIT Num. Math, 50 (2010), 509-539.

[3] F. Dorini, M. C. Cunha, A finite volume method for the mean of the solution of the random transport equation, Appl. Math. Comput, 187 (2) (2007), 912-921.

[4] F. Dorini, M. C. Cunha, Statistical moments of the random linear transport equation, J. Comput. Phys., 227 (2008), 8541-8550.

[5] F. Flandoli, M. Gubinelli, E. Priola, Well-posedness of the transport equation by stochastic perturbation, Invent. Math., 180 (1) (2010), 1-53.

[6] Golub, G. H. and Van Loan, C. F. "Matrix Computations". The Johns Hopkins University Press, 3th Edition, 1996.

[7] L. Grune, P. Kloeden, Pathwise approximation of Random Differential Equations. BIT Num. Math, 41 (2001), 711-721.

[8] H. Kunita, First Order Stochastic Partial Differential Equations, Proceedings of the Taniguchi International Symposium on Stochastic Analysis, 1984.

[9] H. Kunita, "Stochastic flows and stochastic differential equations". Cambridge University Press. 1990.

[10] C. Moler, C.F. Van Loan, Nineteen dubious ways to compute the exponential of a matrix, twenty-five years later, SIAM Review, 45 (2003), 3-49.

[11] H. Osnes, H.P. Langtangen, A study of some finite difference schemes for a unidirectional stochastic transport equation, SIAM J. Sci. Comput., 19 (3) (1998), 799-812. 